\title{
Artefakte in der Computertomografie
}

\section{Artifacts in Computed Tomography}

A. Mayor

Die CT stellt seit ihrer Einführung in den 1970er-Jahren einen wichtigen Bestandteil der medizinischen Diagnostik und Therapie dar. Die Entwicklung führte in den Anfangsjahren hauptsächlich zu größerer Detailgenauigkeit, höherer Auflösung und höherer Geschwindigkeit. Dadurch wurden

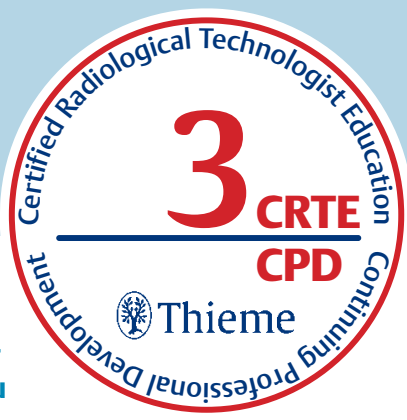
Anfang der 1990er-Jahre CT-Angiografien und mit Mehrzeilen-CT Ganzkörperaufnahmen möglich. Mit dem Fortschritt im IT-Bereich waren auch bessere Nachverarbeitungstechniken möglich. Heute liegt das Hauptaugenmerk bei der Weiterentwicklung auf dem Strahlenschutz für den Patienten. Man möchte mit möglichst kleiner Dosis die besten diagnostischen Bilder erstellen. Jedoch ist dieses Untersuchungsverfahren nicht komplett von Bildstörungen (Artefakten) befreit. Dieser Artikel beschäftigt sich mit der Art von Bildstörungen und was der Anwender machen kann, um diese zu vermindern bzw. zu vermeiden.

\section{Aufbau und Funktionsweise eines Computertomografen}

Die CT ist ein Röntgenverfahren, mit dem transversale Schichten des menschlichen Körpers erzeugt werden können, um die Organe und Gewebe überlagerungsfrei 2-dimensional darzustellen. Um einen Körperabschnitt zu durchstrahlen, rotiert die Röntgenröhre bei gleichzeitiger Tischbewegung (Spiral-CT, $\bullet$ Abb. 1) um den Patienten und sendet einen schmalen fächerförmigen Röntgenstrahl aus. Dieser ist so breit wie die gewünschte Schichtdicke und erfasst den gesamten Körperquerschnitt. Gegenüber der Röntgenröhre befindet sich der Detektor ( $\bullet$ Abb.2). Dieser misst die Intensitätsunterschiede der Röntgenstrahlung und wandelt diese in elektrische Signale um. Die Signale werden dann im Bildrechner verarbeitet, und die gemessenen Schwächungswerte werden mittels gefalteter Rückprojektion in ein Schichtbild umgewandelt.

Der Detektor misst die Intensitätsunterschiede der Röntgenstrahlung und wandelt diese in elektrische Signale um.

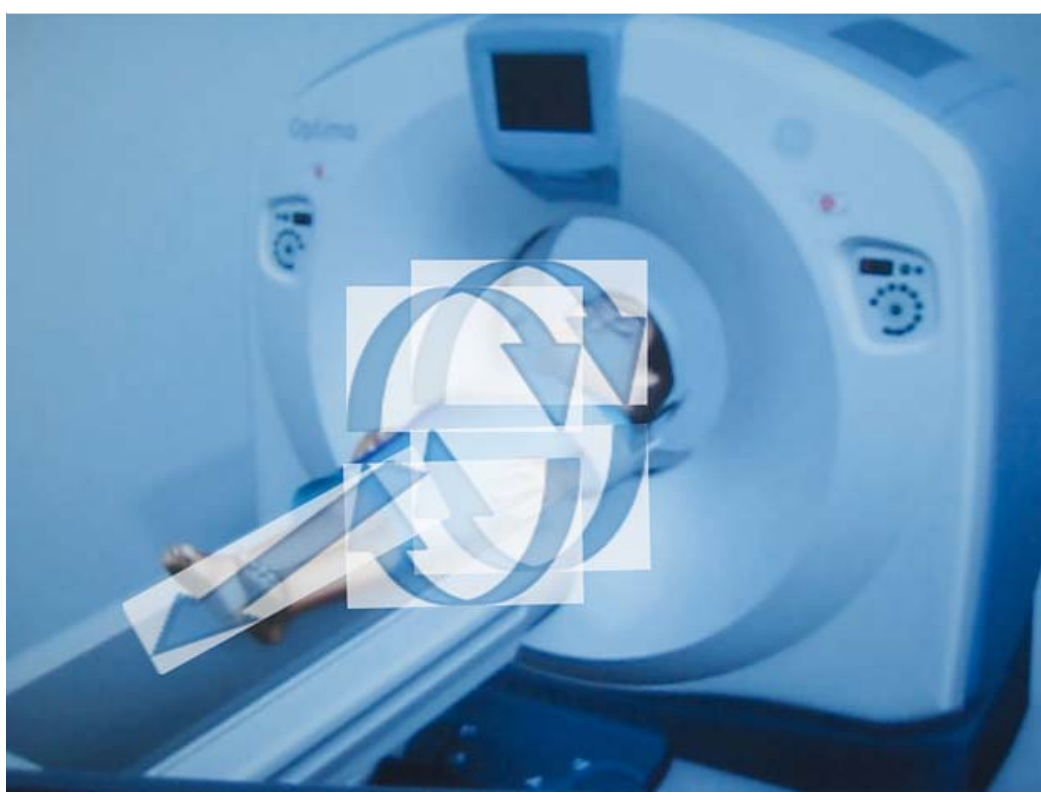

Abb. 1 Spiral-CT.

Weitere Informationen zu Aufbau und Funktionsprinzip finden Sie im Artikel „Computertomografie. Teil 1.“ in Radiopraxis 1/2013. 


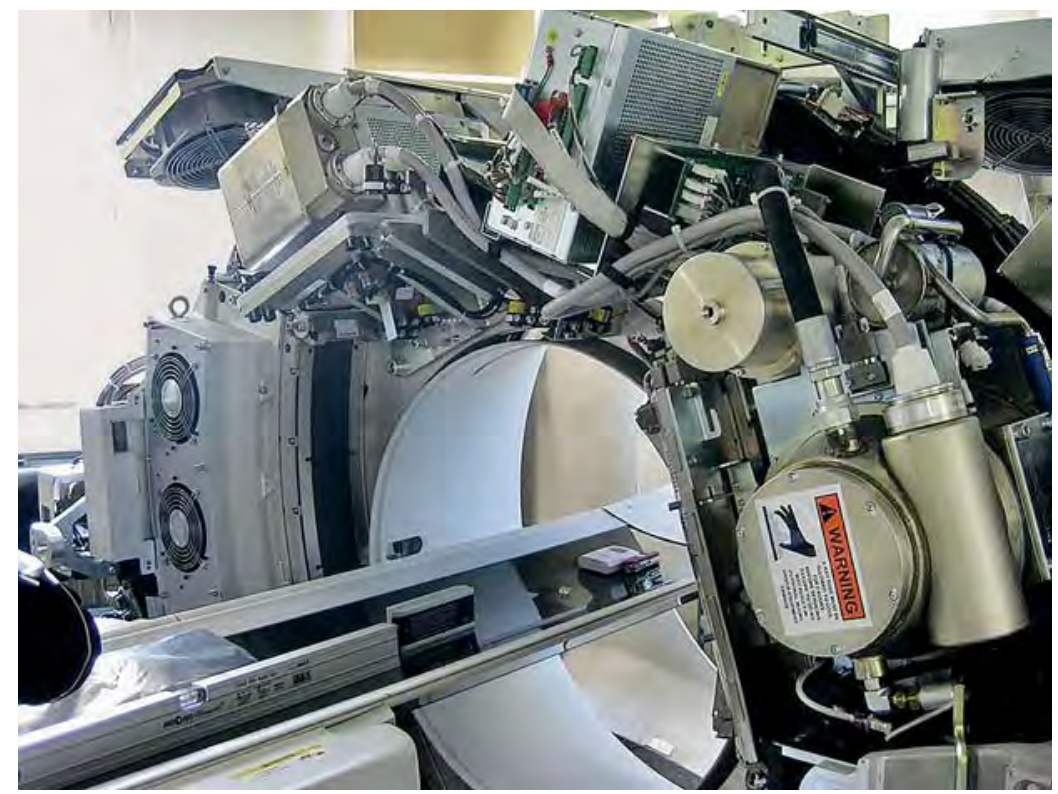

Abb.2 Röntgenröhre (rechts) und Detektorsystem.

\section{Artefakte}

\section{Definition Artefakt $\nabla$}

Als Artefakt (Bildstörung) bezeichnet man ein unechtes, durch Eigenschaften der Methode hervorgerufenes Ergebnis. Diese Bildstörungen können diverse Ursachen haben und können Quellen diagnostischer Unsicherheiten oder Fehlbefundungen darstellen. Daher ist entscheidend, dass die/der befundende Radiologe/in und die/der MTRA in der Lage sind, Artefakte zu erkennen und die Ursache zu beheben.

Artefakte können sich auf verschiedene Weisen darstellen:

streifenförmig

- ringförmig

$\checkmark$ flächenförmig

- linienförmig
- bandförmig

- schattenförmig

verrauscht

spiralförmig

stufenförmig

Patientenverursachte Artefakte. Diese Bildstörungen sind Ergebnisse aus:

- Patientenbewegungen

Atmung

- Herzbewegungen

- dichten Fremdkörpern inner- und außerhalb des Körpers

Bewegungsartefakte. Diese Art von Artefakten lassen sich in

- Körperbewegungsartefakte

- Herzbewegungsartefakte (Pulsationsartefakte)

- Atemartefakte

unterteilen.

Ein Artefakt ist die Diskrepanz zwischen dem abgebildeten Schwächungswert und der tatsächlichen Schwächung im Untersuchungsvolumen.

\section{Körperbewegungsartefakte}

$\nabla$

Die Körperbewegungsartefakte entstehen, wenn sich der Patient während der Datenakquisition bewegt ( $\bullet$ Abb.3). Diese verursachen Streifenund Doppelkonturen im rekonstruierten CT-Bild, die auch ein Ausmaß auf die Bewegung zulassen. Die Beurteilung (z.B. Ausschluss einer Fraktur) von bewegungsbehafteten Daten, vor allem kleinerer Strukturen, wird dadurch extrem erschwert. Im schlimmsten Fall müsste der Scan wiederholt werden (doppelte Strahlenbelastung!).

Die Körperbewegungsartefakte entstehen, wenn sich der Patient während der Datenakquisition bewegt.
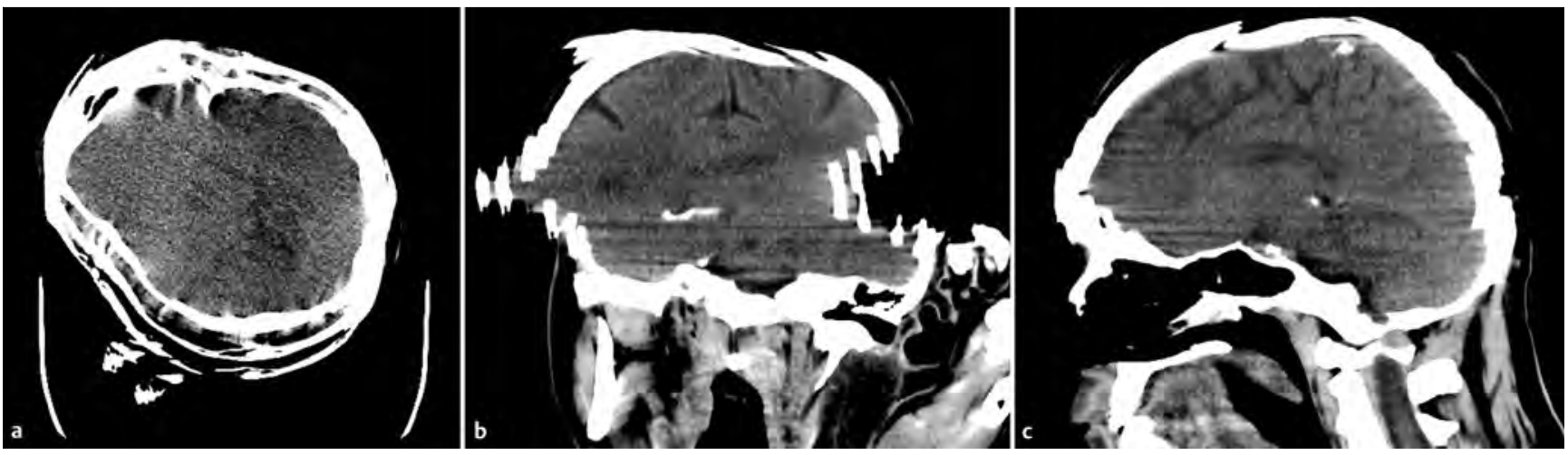

Abb. 3 CCT eines „unruhigen“ Patienten.

a Axial.

b Koronar.

c Sagittal. 


\section{Tipps und Tricks}

Um die Bewegungsartefakte zu vermindern, ist eine gute Vorbereitung und Instruktion des Patienten notwendig. Das Nutzen von Lagerungs-/Fixationshilfen ( Abb.4-6), Minimierung der Scanzeit oder ggf. Sedativa kann zu einer Minimierung führen. Die Scanzeit kann der Nutzer durch Eingrenzung des Scanbereichs, durch Erniedrigung der Rotationszeit oder Erhöhung des Pitchfaktors reduzieren.

\section{Pulsationsartefakte}

$\nabla$

Das schlagende Herz verursacht Pulsationsartefakte. Die Pulsation kann zu Mehrfachkonturen der Aorta thoracalis und/oder unscharfer Abbildung der dem Herzen angrenzenden Organe (z.B. Lunge) führen. Des Weiteren bewirkt dieser Artefakt bei multiplanaren Rekonstruktionen stufenförmige Anhebungen (Zähnelung), vor allem in Patientenlängsrichtung.

Bei der Durchführung von EKG-getriggerten Protokollen können Artefakte ( $\bullet$ Abb.7) auftreten, die durch einen unregelmäßigen bzw. zu schnellen Herzrhythmus hervorgerufen werden, da das System nicht mehr unbedingt in der Lage ist, den Scan zum korrekten Zeitpunkt auszulösen bzw. nach einer gewissen Zeit automatisch den Scan auslöst. Für Kardiountersuchungen sollte eine gewissenhafte Patientenvorbereitung durchgeführt und ggf. Medikamente eingesetzt werden.

Das schlagende Herz verursacht Pulsationsartefakte.

Weitere Informationen zur Kardio-CT finden Sie im Artikel „Herzbildgebung“ in Radiopraxis 2/2013.

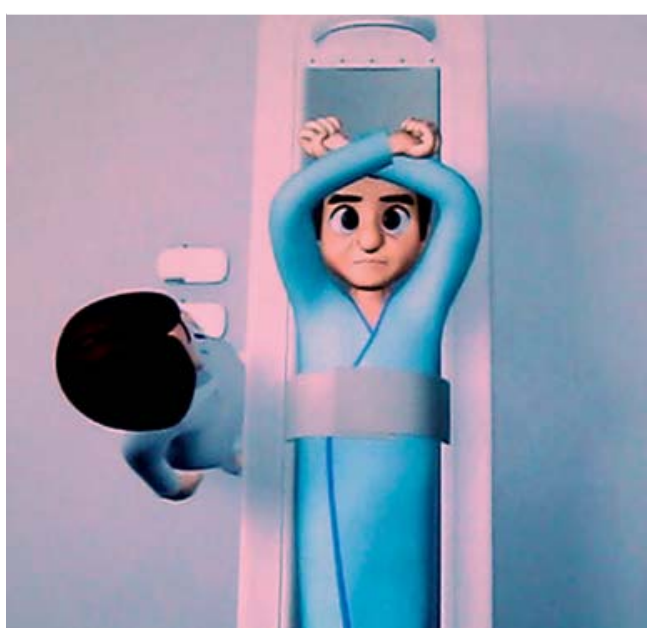

Abb.5 Einsatzmöglichkeit der Fixierbänder.

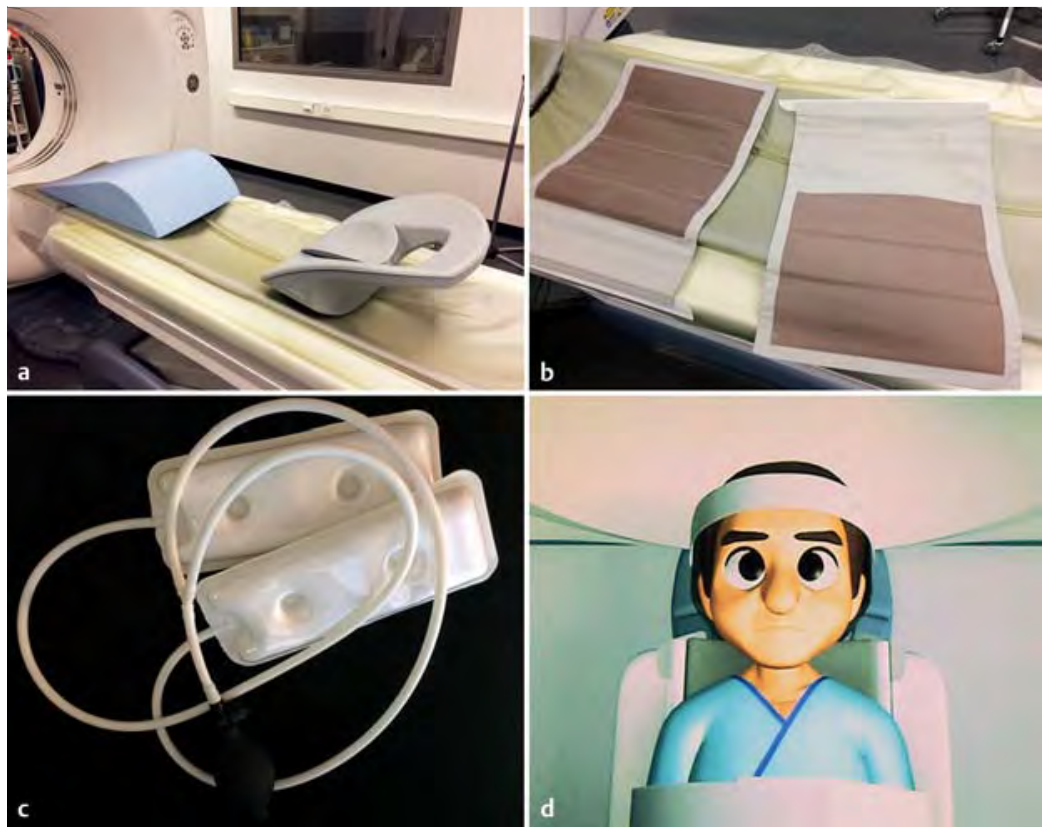

Abb.4 Lagerungshilfen für die CT.

a Kopfhalterung, Kniekissen.

b Fixierbänder.

c Fixierpad.

d Fixierband für Schädeluntersuchungen.

\section{Tipps und Tricks}

Zur Minimierung der Pulsationsartefakte ist eine Reduzierung der gesamten Scanzeit und/ oder Erhöhung der zeitlichen Auflösung zu empfehlen. Auch die Nutzung von EKG-getriggerten Untersuchungsprotokollen ist von Vorteil.
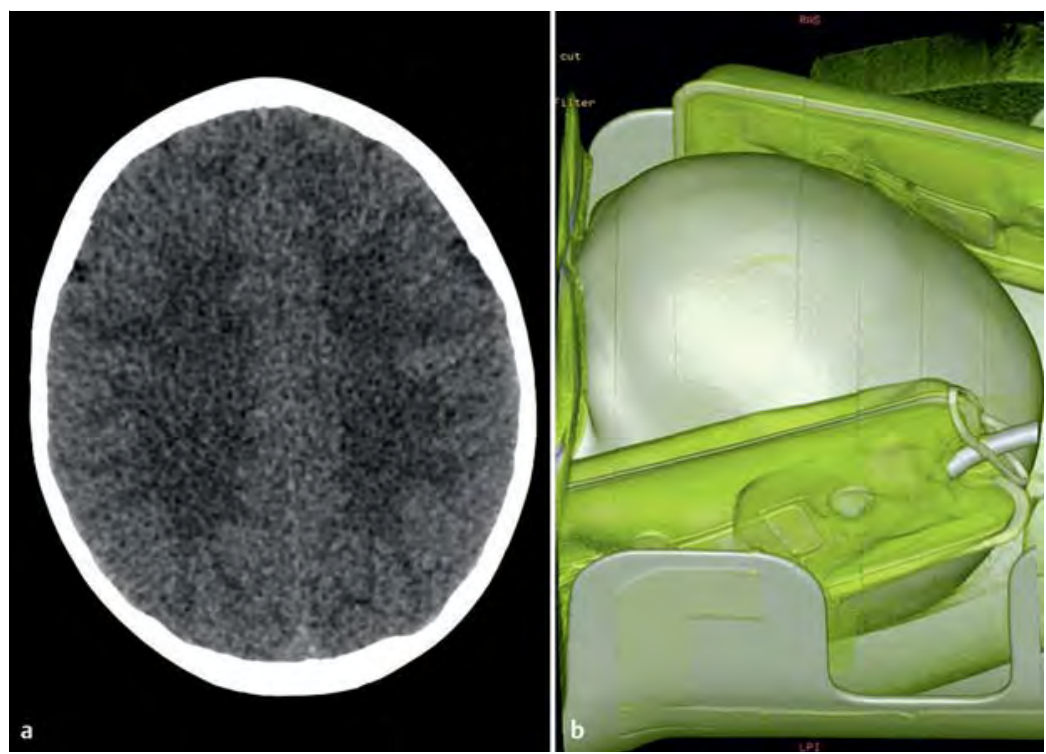

Abb. 6 Einsatz des Fixierpolsters.

a Schnittbild: Das Polster erzeugt keine Bildbeeinträchtigung. b 3-D-Darstellung. 


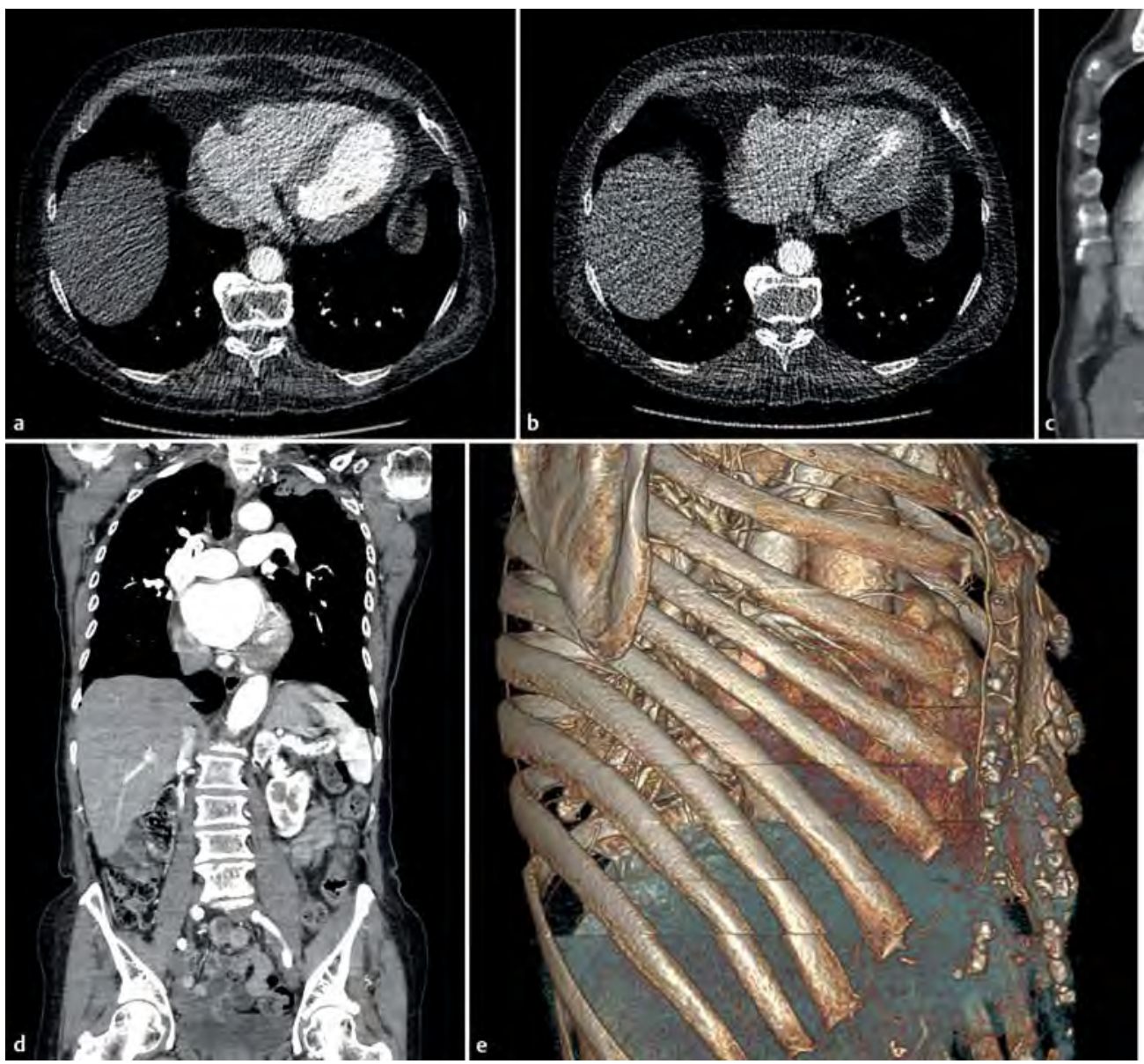

Abb. 7 EKG-getriggertes CT der Aorta.

a, b Zwei aufeinanderfolgende axiale Schichten. Aufgrund des nicht korrekten Scans (EKG-Problem) fehlt Untersuchungsvolumen. c Das EKG-Artefakt stellt sich in der sagittalen Darstellung als Verschiebung der Herzwand bzw. Rippen dar. d Das EKG-Artefakt stellt sich in der koronaren Darstellung als Verschiebung der Herzwand bzw. Rippen dar. e Das EKG-Artefakt stellt sich in der 3-D-Darstellung als Verschiebung der Herzwand bzw. Rippen dar.
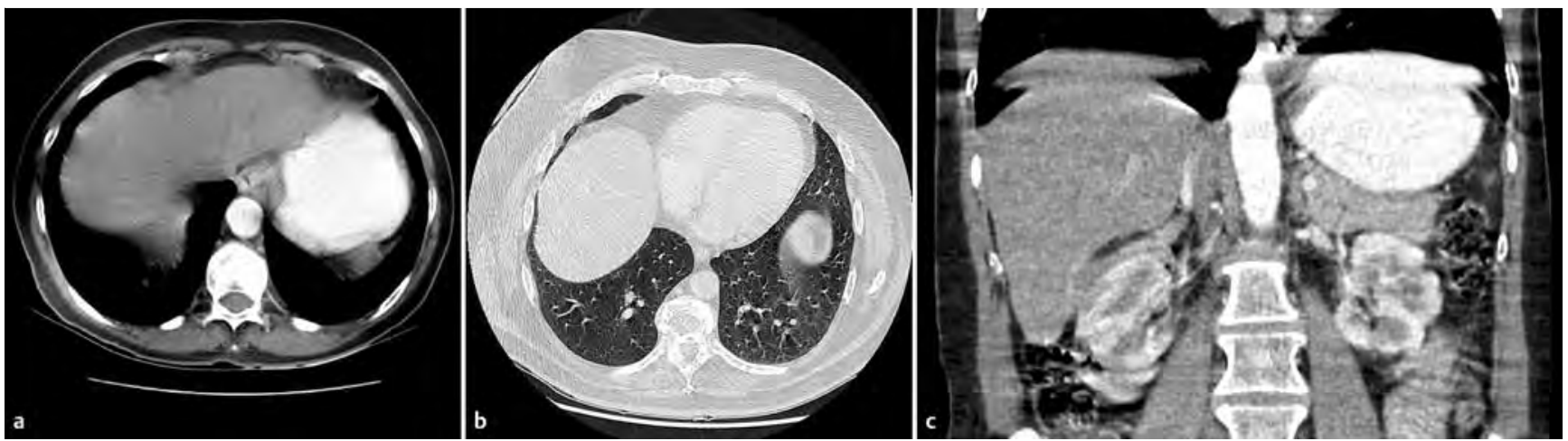

Abb. 8 Beispiel für eine veratmete CT-Thorax-Untersuchung.

a Weichteildarstellung axial.

b Lungenfenster: Die Strukturen sind eingeschränkt beurteilbar.

c Koronare multiplanare Rekonstruktion.

\section{Atemartefakte}

$\nabla$

Diese entstehen durch die Atmung des Patienten. Es kommt durch die Atemverschieblichkeit der Organe zu unscharfen Abbildungen oder Doppelkonturen ( $\bullet$ Abb.8). Sichtbar wird diese Bildstörung durch Mehrfachkonturen der ventralen
Strukturen und welliger Darstellung in multiplanaren Rekonstruktionen. Bei der Durchführung der Untersuchung mit Multislice-CT ist die axiale Bildgebung trotzdem diagnostisch beurteilbar. 


\section{Tipps und Tricks}

Atemkommandos mit dem Patienten vor der Untersuchung üben. Bei beatmeten Patienten sollte ein Atemstopp während des CT-Scans erfolgen (nur nach Absprache mit Anästhesie).

Atemartefakte entstehen durch die Atmung des Patienten.

\section{Metallartefakte}

$\nabla$

Bildstörungen durch metallische Implantate/ Fremdkörper bewirken, dass die Röntgenstrahlung aufgehärtet wird. Dies trifft auch auf Materialien zu, die hauptsächlich Photonen einer bestimmten spezifischen Energie absorbieren. Dadurch werden regelmäßig deutliche Streifen und Schlieren (engl. Streaking) im Bild verursacht. Chirurgische Implantate (z.B. Zahnimplantate, Hüftprothesen o.ä., $\odot$ Abb.9 u. $\odot$ Abb.10) können zur fast vollständigen Absorption mit ausgeprägten Streifenartefakten und vollständigen Überlagerung der Nachbarorgane führen. Kleine Fremdkörper (z.B. Klammernähte) verursachen durch ihr geringes Volumen kaum Artefakte.

Metallartefakte entstehen durch metallische Implantate und metallische Fremdkörper.

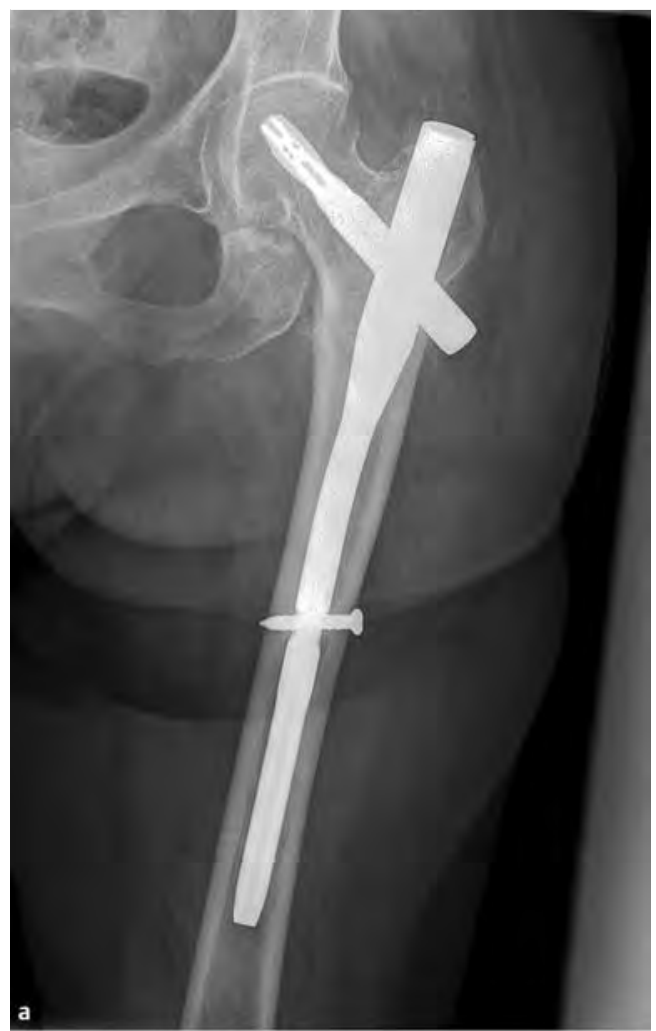

Abb.9 Hüftprothese.

a Konventionelles Röntgenbild. b CT des Beckens.
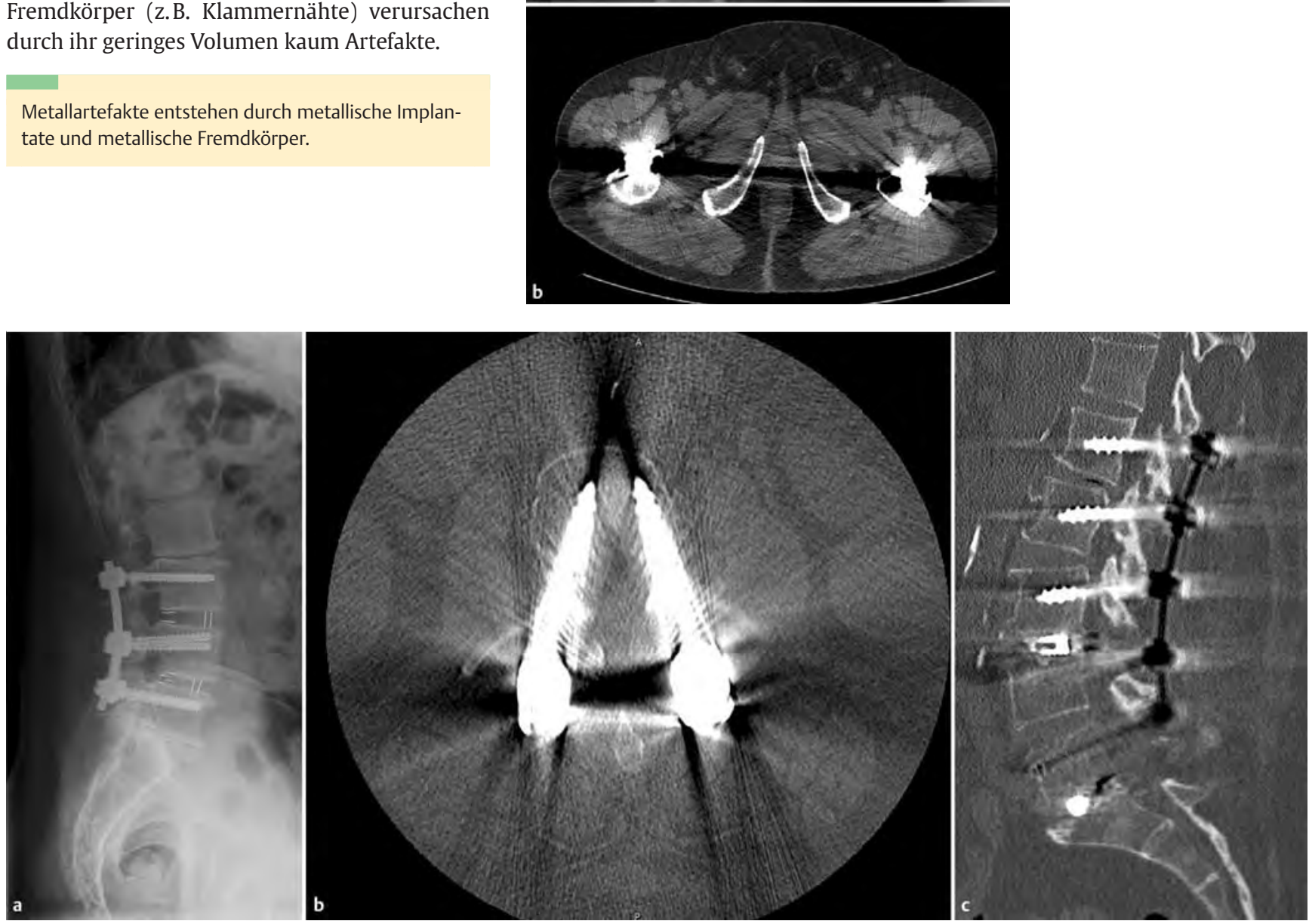

Abb.10 Spondylodese der LWS a Konventionelles Röntgenbild. b CT axial.

c CT multiplanare Rekonstruktion sagittal. 
Abb.11 CT des Beckens. a Scout.

b Ohne Metallreduktionsprogramm. c Mit Metallreduktion.
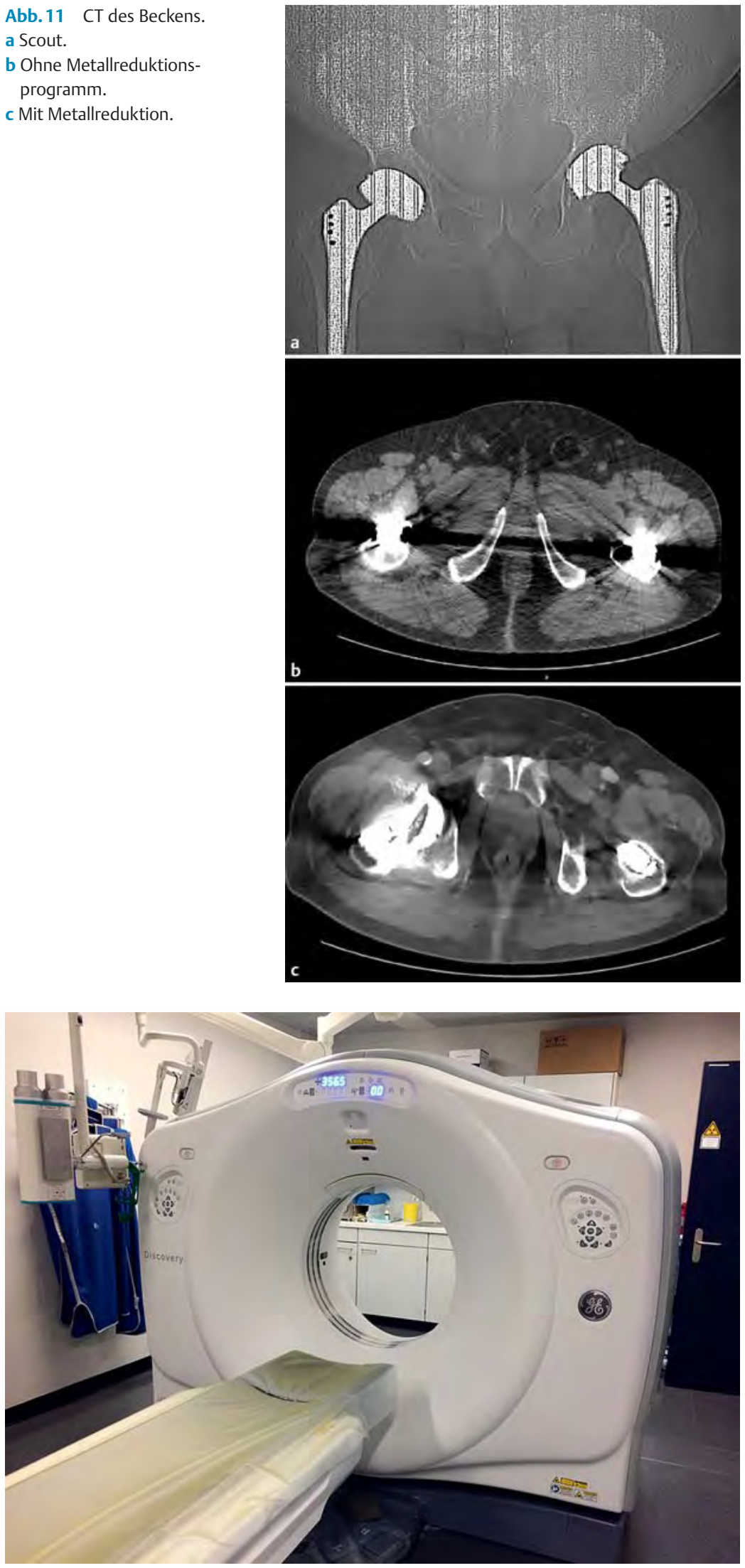

Abb.12 CT mit Spectral Imaging (GE Discovery 750HD).

\section{Tipps und Tricks}

Vor der Untersuchung sollten alle Objekte aus Metall (Zahnprothesen, Schmuck, Sackmesser) aus dem Scanbereich entfernt werden. Falls dies nicht möglich ist (z.B. Osteosynthesen, Hüftprothesen), ist die Verwendung von Metallartefaktreduzierungsprogrammen

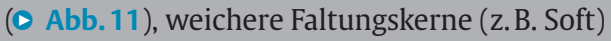
oder die Gantrykippung (beim Schädel) anzuwenden.

\section{Möglichkeiten in der CT zur Metallartefaktreduktion}

\section{Spectral Imaging}

$\nabla$

Dabei werden Scans mit unterschiedlichen Energien $(80 \mathrm{kV}$ und $140 \mathrm{kV})$ angefertigt. Mithilfe der entstandenen unterschiedlichen Energiebilder ( Abb.13) kann man die Gewebe separieren, quantifizieren und materialgewichtete und monochromatische Bilder erzeugen. Monochromatische Bilder bieten eine verbesserte Kontrastmitteldarstellung und Verringerung von Aufhärtungsartefakten ( $\bullet$ Abb.14). Die Szintillationsdetektoren bestehen aus einem besonderen Material: Edelsteinen. Diese haben den Vorteil, dass sie eine hohe Auflösung in der Subsekundenbildgebung bieten und ein viel geringeres Nachglühen besitzen als andere Materialien. Die verschiedenen Bezeichnungen für Spectral Imaging finden sich in $\bullet$ Tab. 1.

Tab. 1 Verschiedene Bezeichnungen für Spectral Imaging.

\begin{tabular}{|l|l|}
\hline Firma & Bezeichnung für Spectral Imaging \\
\hline GE Healthcare & $\begin{array}{l}\text { Gemstone Spectral Imaging } \\
\text { (GSI, } \odot \text { Abb. 12) }\end{array}$ \\
\hline $\begin{array}{l}\text { Siemens } \\
\text { Philips }\end{array}$ & Spectral Imaging \\
\hline
\end{tabular}

Das niedrige Nachglühen der Szintillationsdetektoren führt zu einer erhöhten räumlichen Auflösung und wenig Artefakten.

\section{Iterative Rekonstruktionstechnik}

Das iterative Rekonstruktionsverfahren wurde 2010 auf dem Markt eingeführt und wird zunehmend zum Standard in den neuesten CT-Scannern. Für das Verfahren benötigt man schnellere Bildrechner, da die Berechnung mit der vorhandenen Technik länger dauern kann. Da dieses Verfahren rein rechnerisch stattfindet, hat es folgende Vorteile: 

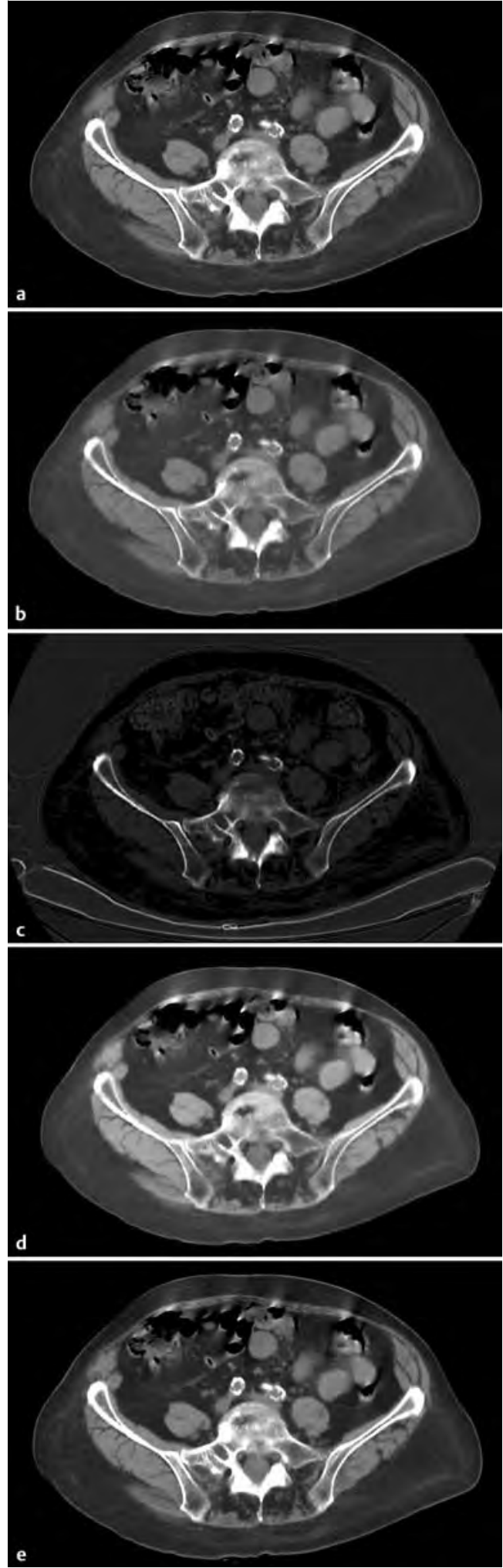

Abb. 13 Bildbeispiel für Spektral Imaging. a $80 \mathrm{kV}$.

b $140 \mathrm{kV}$.

c Jod-Bild.

d Wasserbild.

e Monochromatisches Bild mit $70 \mathrm{keV}$.
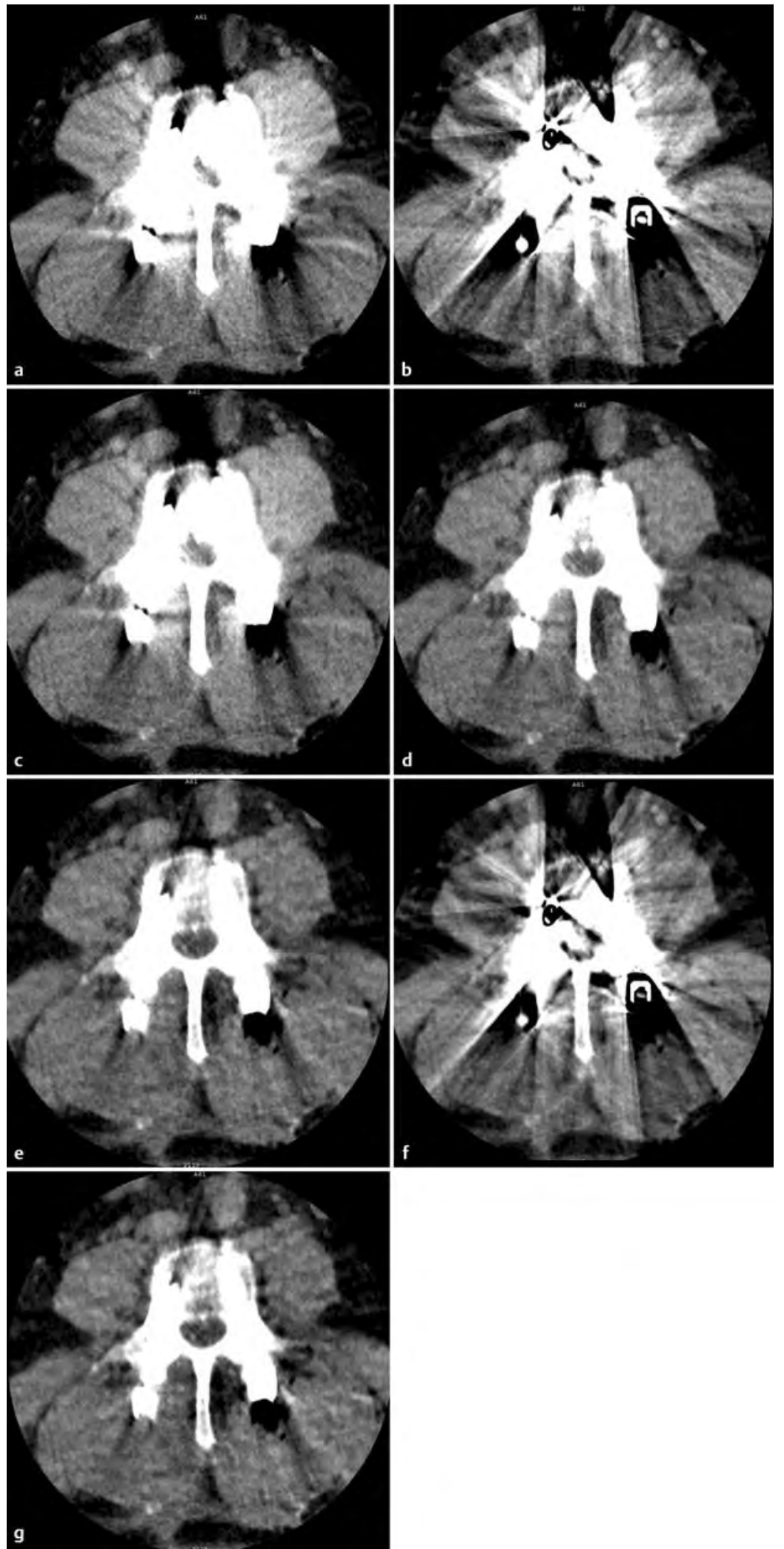

Abb.14 Bildbeispiel für Spektral Imaging mit und ohne Metallreduktion (MARS).

a $70 \mathrm{keV}$.

b $80 \mathrm{keV}$.

c $80 \mathrm{keV}$ mit MARS.

d $100 \mathrm{keV}$ mit MARS.

e $120 \mathrm{keV}$ mit MARS.

f $140 \mathrm{keV}$.

g $140 \mathrm{keV}$ mit MARS. 
- Verbesserung des Bildrauschens, Artefaktreduktion bei gleicher Dosis: iterative Rekonstruktion im Bilddatenbereich

- Reduzierung der Dosis bei gleichem Bildeindruck wie bei der gefilterten Rückprojektion (Filtered back Projection, FBP): iterative Rekonstruktion in den Rohdaten

- Kombination aus beiden: iterative Rekonstruktion im Bild-/Rohdatenbereich ( $\bullet$ Abb. 15)

Im Gegensatz zur gefilterten Rückprojektion wird eine Korrekturschleife in den Bilderzeugungsprozess eingefügt. Die Projektionsdaten werden iterativ (lat. iterare $=$ wiederholen) mit den eigentlichen Messdaten verglichen. Das Bild wird durch ein Korrekturbild aktualisiert, und Vorwissen (Rauschcharakteristik der Röntgenphotonen) fließt in die Bilddaten ein. Durch das Vorwissen wird das Bild innerhalb homogener Bereiche geglättet, während die Kontrastgrenzen bleiben. Im korrigierten Bild ist die räumliche Auflösung bei erhöhtem Objektkontrast verbessert und das
Bildrauschen in kontrastarmen Bereichen verringert. Da das Verfahren die Bilddaten mit den Originalmessdaten vergleicht, kann mithilfe der iterativen Rekonstruktion die Strahlenexposition des Patienten (um bis zu 60\%) erheblich gesenkt werden, ohne auf den gewohnten Bildeindruck verzichten zu müssen.

Das iterative Rekonstruktionsverfahren wird zunehmend zum Standard in den neuesten CT-Scannern.

Weitere Informationen zur iterativen Rekonstruktion finden Sie im Artikel „Computertomografie. Teil 1.“ in Radiopraxis 1/2013.

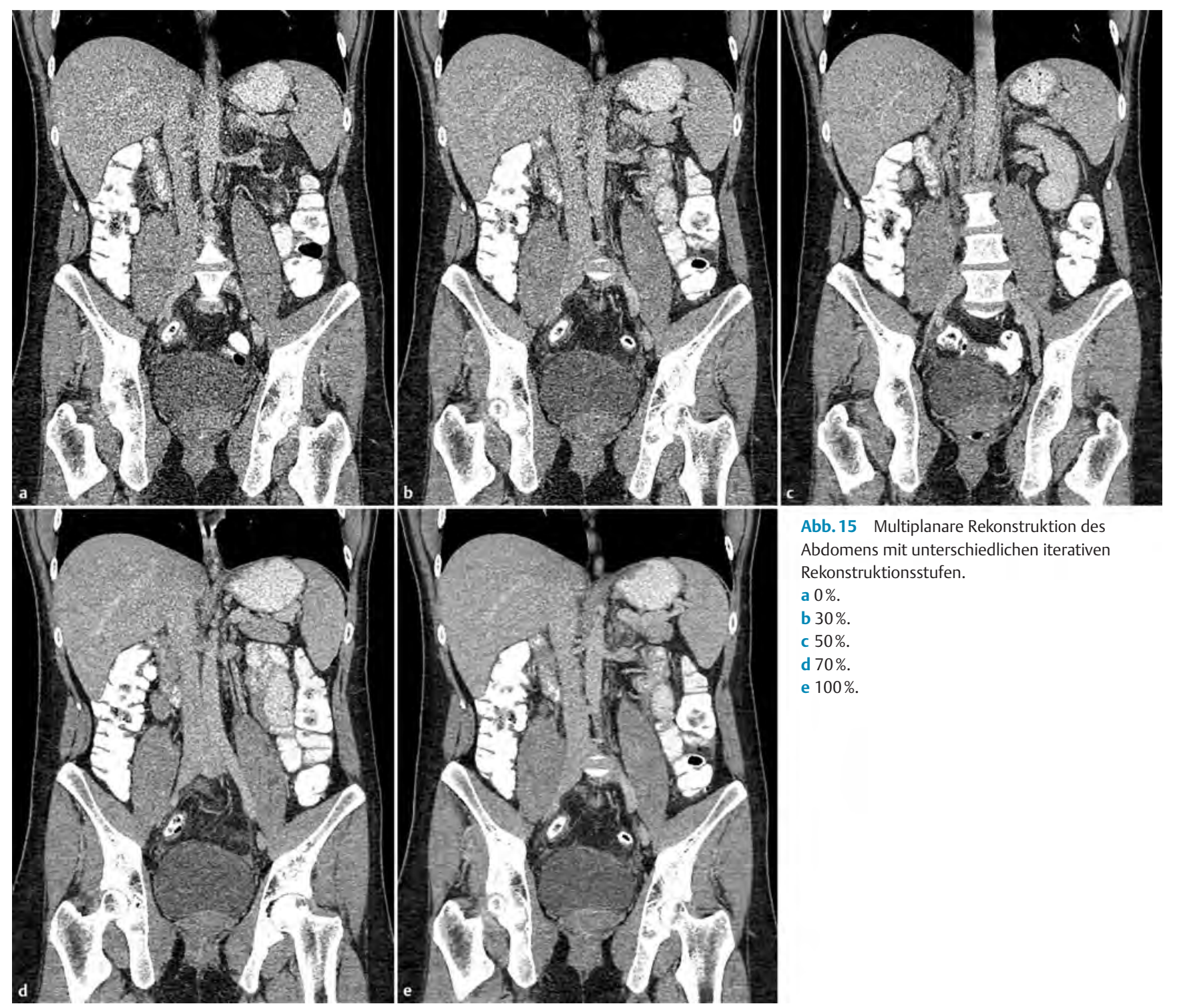



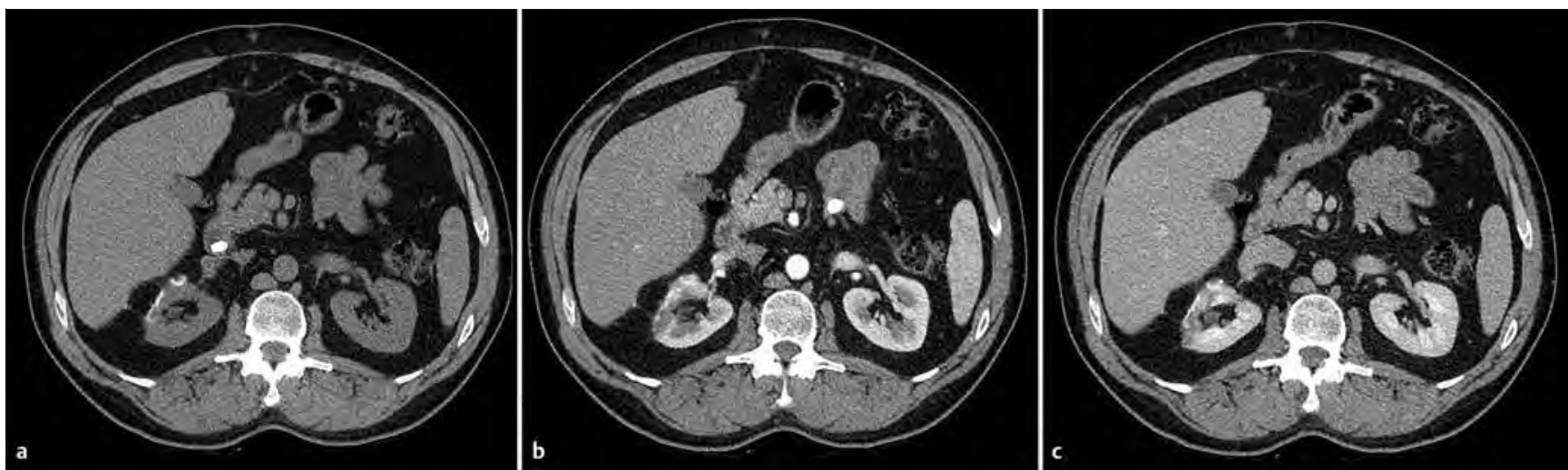

Abb. 16 Mehrphasiges CT des Abdomens. Verlaufskontrolle bei Zustand nach Nierenteilresektion bei Onkozytom mit entsprechenden postoperativen narbigen Veränderungen.

a Nativ.

b Arteriell.

c Venös.

\section{Anatomie- und pathologie- bedingte Artefakte}

\section{Anatomische Lagevarianten \\ $\nabla$}

Die nicht genaue Abgrenzung zwischen Geweben (Venenlymphknoten, Darmgefäßlymphknoten) kann eine Läsion vortäuschen. Um die Läsion zu verifizieren, ist die Betrachtung in einer 2. Ebene mit i.v.-Kontrastmittelapplikation hilfreich.

\section{Pathologiebedingte Artefakte}

$\nabla$

Nach Operationen (z.B. Bandscheibenvorfall) kann das nicht eindeutig identifizierte Gewebe (Narbe, Rezidiv) eine Läsion darstellen. Die Unterscheidung erfolgt mithilfe einer i.v.-Kontrastmittelapplikation ( $\bullet$ Abb.16).

\section{Physikalische Artefakte}

Physikalische Artefakte resultieren aus dem Messprozess der Datenakquisition.

\section{Aufhärtungsartefakte \\ $\nabla$}

Das Röntgenspektrum besteht aus Photonen unterschiedlicher Energie. Niederenergetische Photonen werden im Vergleich zu höherenergetischen Photonen im Gewebe stärker absorbiert, wodurch auch das Röntgenspektrum so geändert wird, dass am Detektor verhältnismäßig mehr höherenergetische Photonen ankommen als niederenergetische. Dieser Vorgang nennt sich Strahlungsaufhärtung und kann zu Störungen des rekonstruierten Datensatzes führen.

Aufhärtungsartefakte stellen sich als Streifenartefakte oder sog. Cupping dar. Man bezeichnet damit den Effekt, dass das gemessene Schwächungsprofil vom idealen und wahren Profil abweicht. Der Grund dafür ist, dass das Schwächungsprofil

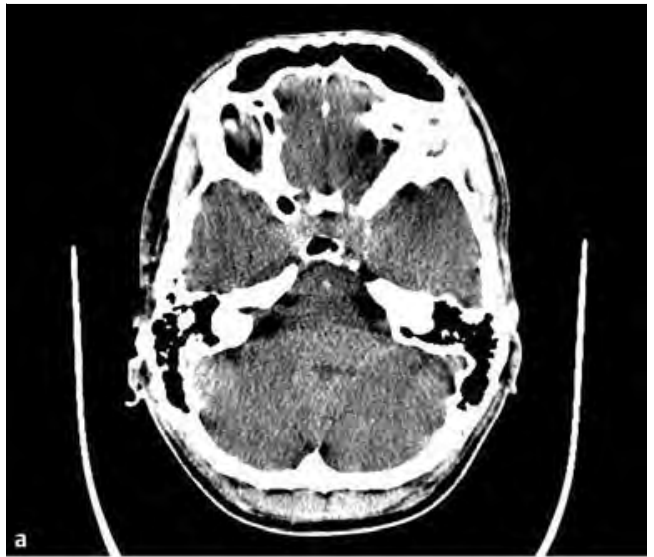

Abb.17 Aufhärtungsartefakt. a Beim Schädel-CT. b Bariumkontrastmittel.

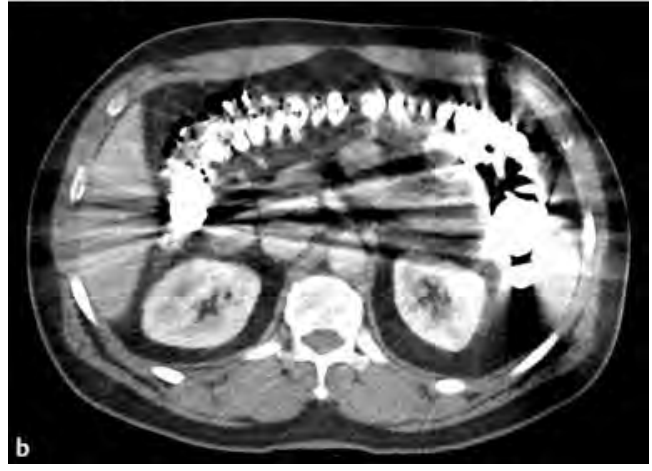

eines Materials energieabhängig ist. Daher besitzt ein Volumenelement des Materials in größerer Objekttiefe einen geringeren Schwächungskoeffizienten. Auch an Geweben mit stark unterschiedlichen Schwächungswerten (z.B. Knochen und Luft, Schädelbasis, $\bullet$ Abb.17a) oder bei Verwendung von unverdünntem Bariumkontrastmittel $(\bullet$ Abb.17b) treten solche Aufhärtungsartefakte auf.

Aufhärtungsartefakte stellen sich als Streifenartefakte dar. Man bezeichnet damit den Effekt, dass das gemessene Schwächungsprofil vom idealen und wahren Profil abweicht. 


\section{Tipps und Tricks}

Um dieser Bildstörung entgegenzuwirken, werden verschiedene Filter angewendet. Der niederenergetische Anteil wird durch einen Vorfilter an der Röntgenröhre absorbiert. Ein weiterer Filter härtet das Spektrum an der Peripherie des Untersuchungsvolumens auf, wo das zu scannende Patientenvolumen geringer ist. Für den Betrachter kann die Nachrekonstruktion der Daten mit einer größeren Schichtdicke ausreichend sein. Des Weiteren ist die regelmäßige Kalibration des CT-Systems durchzuführen und der Einsatz der iterativen Rekonstruktionstechnik zu bedenken.

\section{Partialvolumenartefakte \\ $\nabla$}

Diese Bildartefakte entstehen, wenn im gleichen Voxel Materialien mit unterschiedlichen Absorptionskoeffizienten abgebildet werden. Ursache: Auf einen Detektor trifft ein Strahl mit dem Schwächungskoeffizienten $\mu_{1}$ und ein Strahl mit dem Koeffizienten $\mu_{2}$. Dieser registriert eine Mittelung aus beiden über der verwendeten Kollimation (und/oder Schichtdicke). Dieser vom Computer generierte Mittelwert entspricht nicht dem realen Dichteprofil der darzustellenden Struktur. Dieses Artefakt ist von Bedeutung bei Rekonstruktionen mit großer Schichtdicke, da es eine Läsion vortäuschen kann. Ebenfalls spricht man von Partialvolumeneffekten, wenn ein Objekt, welches sich nicht im Isozentrum des Scanners befindet, mit nicht genügend Messdaten (Projektionen) zur Bildrekonstruktion beiträgt. Die Ursache liegt daran, dass das Objekt aufgrund der Divergenz der Röntgenstrahlung nur partiell abgetastet wird. Der Effekt zeigt sich als Schatten im Bild.

Partialvolumenartefakte entstehen, wenn im gleichen Voxel Materialien mit unterschiedlichen Absorptionskoeffizienten abgebildet werden.

\section{Photon Starvation \\ $\nabla$}

Das Photon-Starvation-Artefakt (engl. Starvation =Das Verhungern) ist definiert durch ein verstärktes Bildrauschen in bestimmten Bildanteilen, welches durch eine erhöhte Abschwächung der Röntgenstrahlung durch Unterschiede in der Morphologie verursacht wird. Im Untersuchungsvolumen treffen auf den Detektor aus unterschiedlichen Projektionen weniger Photonen (z.B. stärkere Schwächung durch die Schulter, - Abb.19a). Dies führt zu streifigen Bildanteilen

\section{Tipps und Tricks}

Der Partialvolumeneffekt ( $\bullet$ Abb. 18) kann reduziert werden, wenn man dünne Schichtdicken verwendet und die Projektionen erhöht. Die Daten sollten zudem in verschiedenen Schichtebenen betrachtet werden. Vor allem bei kontrastreichen Gewebsabschnitten (z.B. Lunge) ist die Schichtdicke möglichst klein zu wählen.

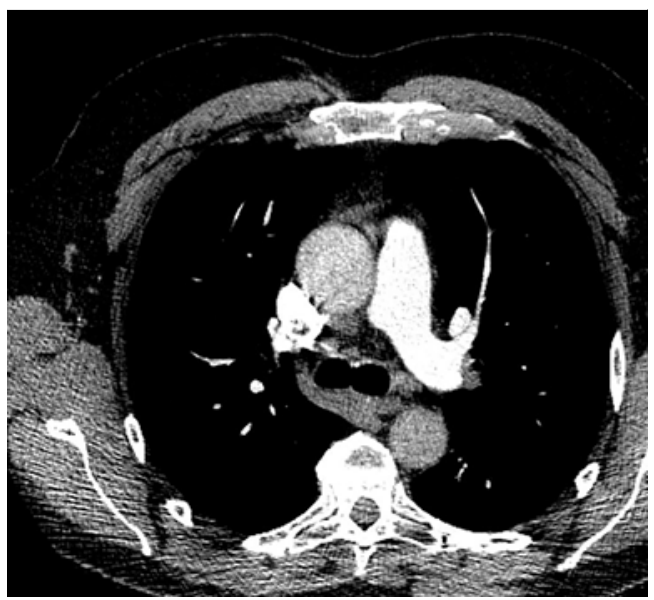

Abb.18 Partialvolumeneffekt.

mit erhöhtem Bildrauschen. Falls die Arme während einer Thorax-Abdomen-Untersuchung nicht über den Kopf gelagert werden können, sollten diese besser ventral statt neben den Körper gelagert werden. Der Röhrenstrom muss dann angepasst werden, um das zusätzliche Durchstrahlungsvolumen auszugleichen ( $\bullet$ Abb.19b).
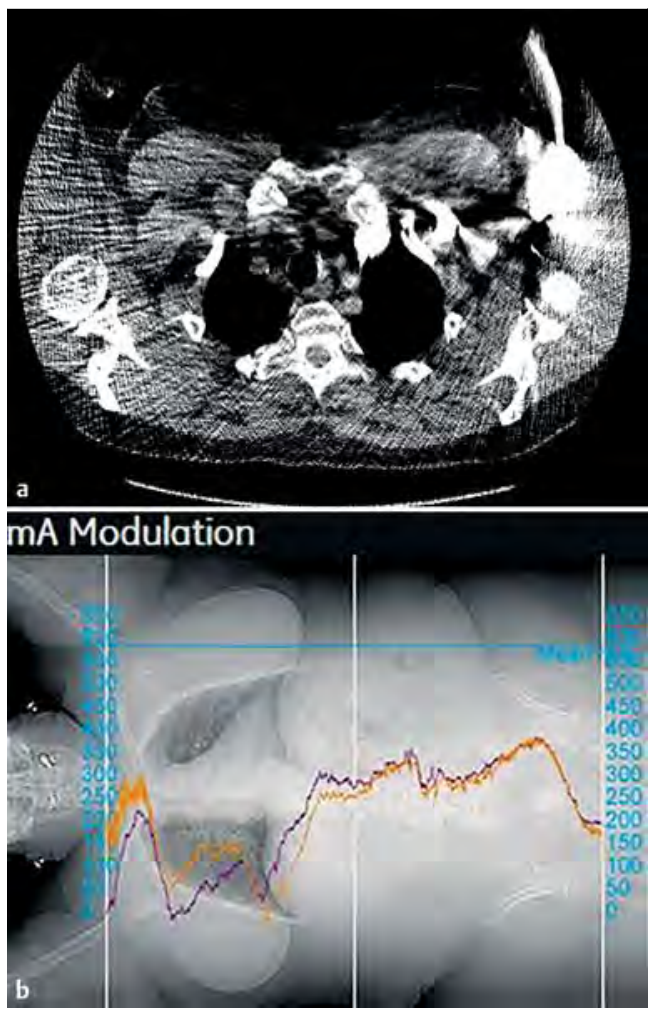

Abb.19 Photon Starvation.

a Starkes Bildrauschen in Schulterhöhe. b Beispiel für Dosismodulation. 
Das Photon-Starvation-Artefakt zeigt sich durch ein verstärktes Bildrauschen in bestimmten Bildanteilen, das durch eine erhöhte Abschwächung der Röntgenstrahlung durch Unterschiede in der Morphologie verursacht wird.

\section{Tipps und Tricks}

Durch die schwächungswertbasierte Modulation des Röhrenstroms, iterative Rekonstruktion und/oder adaptive Filterung kann eine Erniedrigung der Artefakte erreicht werden.

\section{Unterabtastung}

$\nabla$

Als Unterabtastung (engl. Undersampling) bezeichnet man die zu geringe Aufnahme oder Verwendung von Projektionsdaten, die zu artefaktfreien Rekonstruktionen benötigt werden. Wird das zu untersuchende Objekt mit zu wenigen oder zu großen Winkelschritten gemessen, treten sogenannte Aliasing-Effekte ( $\bullet$ Abb.20) auf. Diese führen zur Entstehung von feinen, potenziell gitternetzartigen Linien in unmittelbarer Nähe von Objektkanten. Artefakte dieser Art werden verursacht, wenn das im Scanner befindliche Untersuchungsvolumen größer ist (z.B. Adipositas, - Abb.21) oder sich außerhalb (z.B. i.v.-Zugang für Kontrastmittelapplikation) des Scan-Field-ofView (Scan-FOV) befindet. Dieses macht Artefakte, ist aber auf dem rekonstruierten Bild nicht sichtbar.

Als Unterabtastung bezeichnet man die zu geringe Aufnahme oder Verwendung von Projektionsdaten, die zu artefaktfreien Rekonstruktionen benötigt werden.

\section{Tipps und Tricks}

Der Patient wird innerhalb des Scan-FOV gelagert, und die Rotationsgeschwindigkeit und Pitch werden reduziert.

\section{CT-basierte Artefakte}

CT-basierte Artefakte entstehen durch Ungenauigkeiten oder Fehler in der Messtechnik oder im System.

\section{Ringartefakte \\ $\nabla$}

Ringartefakte entstehen entweder aus der fehlerhaften Funktion (Temperaturschwankungen, Alter des Detektors) oder der mangelnden Kalibrierung der Detektoreinheiten zueinander. Die

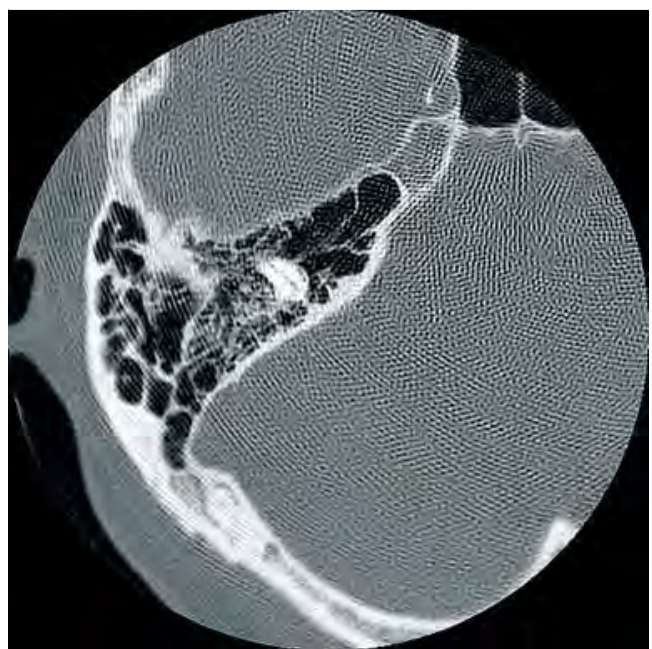

Abb. 20 Aliasing-Artefakt: Innenohr.

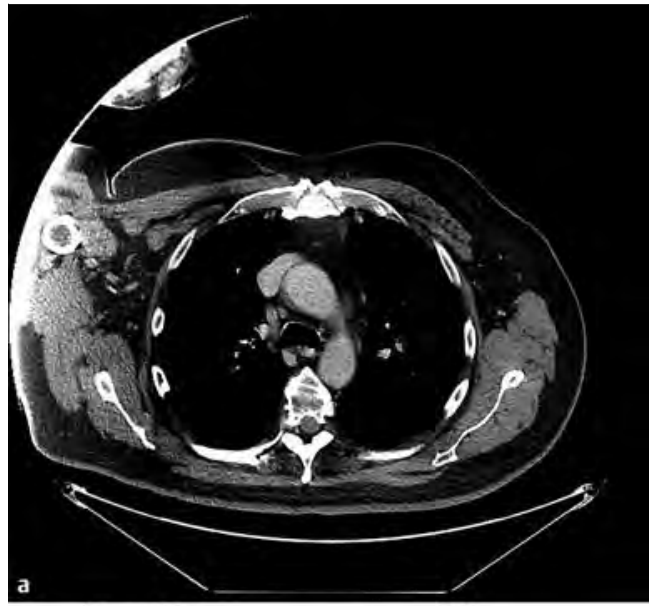

Abb.21 Bildstörungen durch Adipositas.

a Axial.

b Koronar.

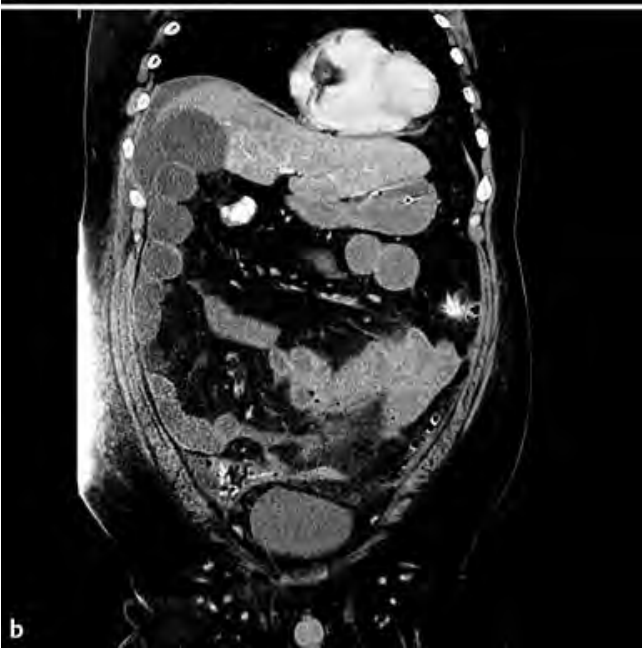

im CT-Bild exakt kreisförmigen erkennbaren Bildstörungen kommen durch die beständigen Fehlmessungen an jeder Winkelposition zustande. Die Mittelpunkte der Artefakte sind kongruent zur Rotationsachse des Scanners. Die heutigen installierten Systeme besitzen eine hohe Empfindlichkeit sowie eine hohe räumliche Auflösung. Diese Vorteile erhöhen aber die Wahrscheinlichkeit für das Auftreten dieses technischen Artefakts $(\bullet$ Abb.22). 
Abb.22 Ringartefakt (Phantommessung).

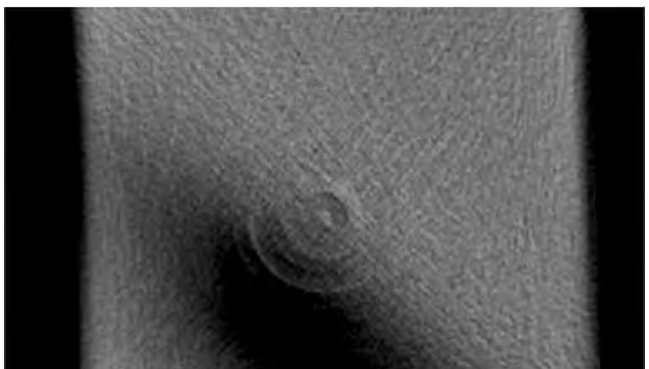

Ringartefakte entstehen entweder aus der fehlerhaften Funktion oder der mangelnden Kalibrierung der Detektoreinheiten zueinander.

\section{Tipps und Tricks}

- Das System sollte regelmäßig nach Vorgaben der Herstellerfirma kalibriert werden.

- Temperaturschwankungen sollten vermieden werden.

- Bei weiterem Auftreten sollte der Service kontaktiert werden.

\section{Spiralartefakte \\ $\nabla$}

Die Art von Bildstörungen haben ihre Ursachen in der Tatsache, dass für die Bildrekonstruktion im Spiral-CT eine Interpolation der Messdaten notwendig ist. Das System interpoliert die mittels unterschiedlicher Detektorsysteme gemessenen Projektionsdaten in Patientenlängsachse (z-Achse) auf identischen Tischpositionen. Spiralartefakte stellen sich als windmühlenartige, regelmäßig radial verlaufende Hell-Dunkel-Streifen dar. Wenn der Bediener die axialen Bilder schnell „durchscrollt“, erscheinen diese ihm als rotierend. Diese Bildstörung tritt vor allem bei Strukturen mit hohem Kontrast auf (z.B. Schädelbasis).

Spiralartefakte stellen sich als windmühlenartige, regelmäßig radial verlaufende Hell-Dunkel-Streifen dar.

Tab. 2 Weitere Fehlermöglichkeiten.

\begin{tabular}{|l|l|}
\hline Fehler & Auswirkung \\
\hline Röhrendefekt & $\begin{array}{l}\text { Der Scan wird durch das System abgebrochen. Die Bilder } \\
\text { stellen sich als stark verrauscht bzw. nur als Rauschen } \\
\text { dar. Hier muss die Untersuchung abgebrochen und der } \\
\text { Service informiert werden. }\end{array}$ \\
\hline Detektorausfall & $\begin{array}{l}\text { Ein Ausfall zeigt sich als schwarze oder weiße Linie im } \\
\text { Bild. Auch hier ist der Service zu kontaktieren. }\end{array}$ \\
\hline Hochspannungsproblem & $\begin{array}{l}\text { Verursacht durch Störungen in der Hochspannung } \\
\text { (- Abb. 23). }\end{array}$ \\
\hline Rekonstruktionsartefakte & $\begin{array}{l}\text { Unter dem Begriff versteht man Bildstörungen, die } \\
\text { durch den Prozess der Bildnachverarbeitung entstehen. }\end{array}$ \\
\hline $\begin{array}{l}\text { Stufenbildung bei multi- } \\
\text { planarer Rekonstruktion, } \\
\text { 3-D }\end{array}$ & $\begin{array}{l}\text { Die Stufen } \\
\text { planaren Rekonstruktion Daten mit einer zu großen } \\
\text { Schichtdicke, keine Überlappung oder Daten aus einer }\end{array}$ \\
\hline sequenziellen Aufnahme verwendet werden.
\end{tabular}

\section{Tipps und Tricks}

Die heutigen CT-Systeme haben Algorithmen in der Bildrekonstruktion, die dieses Artefakt minimieren (z. B. z-Sharp, Double-z-Sampling, IQ Enhance). Des Weiteren hilft die Rekonstruktion von dickeren Schichten aus primär dünneren Scans, das Auftreten zu reduzieren. Jedoch sinkt mit der größeren Schichtdicke die Auflösung in die Patientenlängsrichtung. Einen Kompromiss bildet die Auswahl des Rekonstruktionsinkrements. Die optimale Überlappung liegt zwischen $>0,5$ und $<1,0$ der verwendeten Detektorkollimation (Beispiel: Bei einer Kollimation von 0.625 mm sollte die Überlappung für die Bilddaten zwischen $>0.312$ und $<0.625$ betragen.) Alternativ steht vor allem bei Schädeluntersuchungen der sequenzielle Scan zur Verfügung.

\section{Kegelstrahlartefakte \\ $\nabla$}

Kegelstrahlartefakte (engl. Cone Beam Artifacts) entstehen durch die Geometrie des Röntgenfächers bei Multislice-CT. Bei der Verwendung von mehreren Detektoren divergieren die Röntgenstrahlen beim Austritt aus der Röntgenröhre auf ihrem Weg durch die Gantry. Jedoch wird bei der Bildrekonstruktion durch die gefilterte Rückprojektion (engl. Filtered Back Projection) näherungsweise eine Parallelität der Strahlung angenommen. Die tatsächlich vorhandene Divergenz der Strahlung verursacht eine geometrische Verzerrung im gemessenen Voxel, welche mit Entfernung zum Rotationszentrum zunimmt. Die Artefakte ähneln denen des Partialvolumeneffekts und finden sich hauptsächlich fern des Isozentrums. Weitere Fehlermöglichkeiten sind in - Tab. 2 dargestellt.

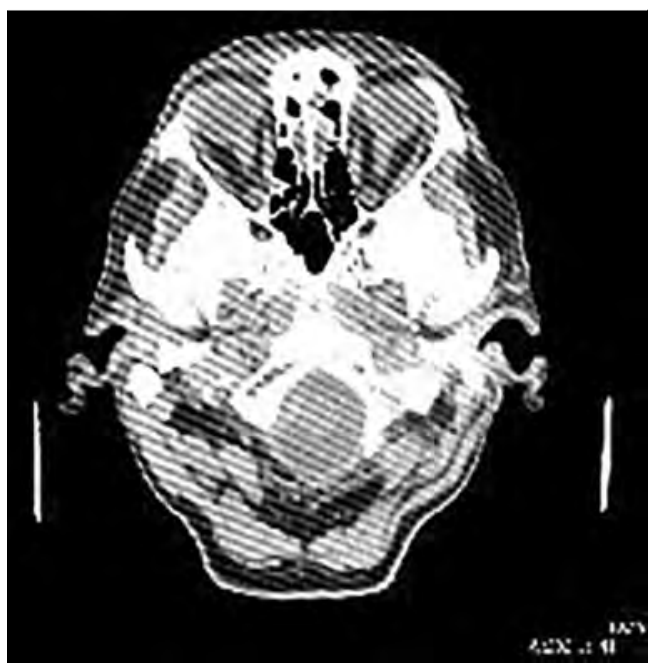

Abb.23 Beispiel für ein Hochspannungsproblem. 

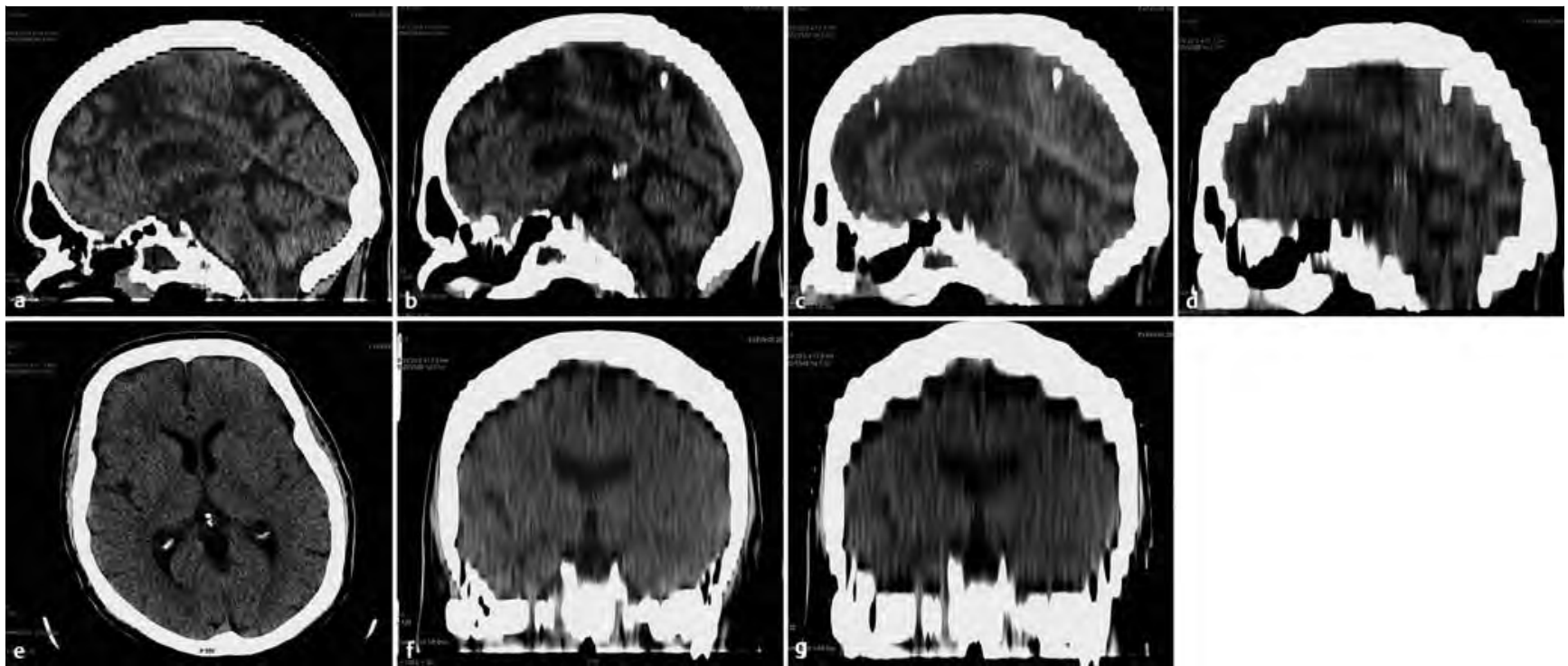

Abb.24 Multiplanare Rekonstruktionen mit unterschiedlicher Schichtdicke.

a $2,5 \mathrm{~mm}$.

b $3,75 \mathrm{~mm}$.

c $5 \mathrm{~mm}$.

d $5 \mathrm{~mm}$ Schichtdicke, $10 \mathrm{~mm}$ Abstand.

e $5 \mathrm{~mm}$ axial.

f $5 \mathrm{~mm}$ koronar.

g $5 \mathrm{~mm}$ Schichtdicke, $10 \mathrm{~mm}$ Abstand koronar.

Je mehr Zeilen ein CT-System besitzt, desto stärker ist die geometrische Verzerrung und dadurch auch die

Ausprägung der Kegelstrahlartefakte.

\section{Tipps und Tricks}

Der Nutzer kann in der Regel keinen Einfluss auf eine Reduzierung der Kegelstrahlartefakte nehmen. Die Firmen verwenden Cone-BeamRekonstruktionsalgorithmen zur Kompensation.

\section{Tipps und Tricks}

Es sollten Daten verwendet werden, die mittels dünner Kollimation und Überlappung erstellt wurden.

Weitere Informationen zur Nachverarbeitung in der Computertomografie finden Sie im Artikel „Computertomografie. Teil 2.“ in Radiopraxis $4 / 2013$.
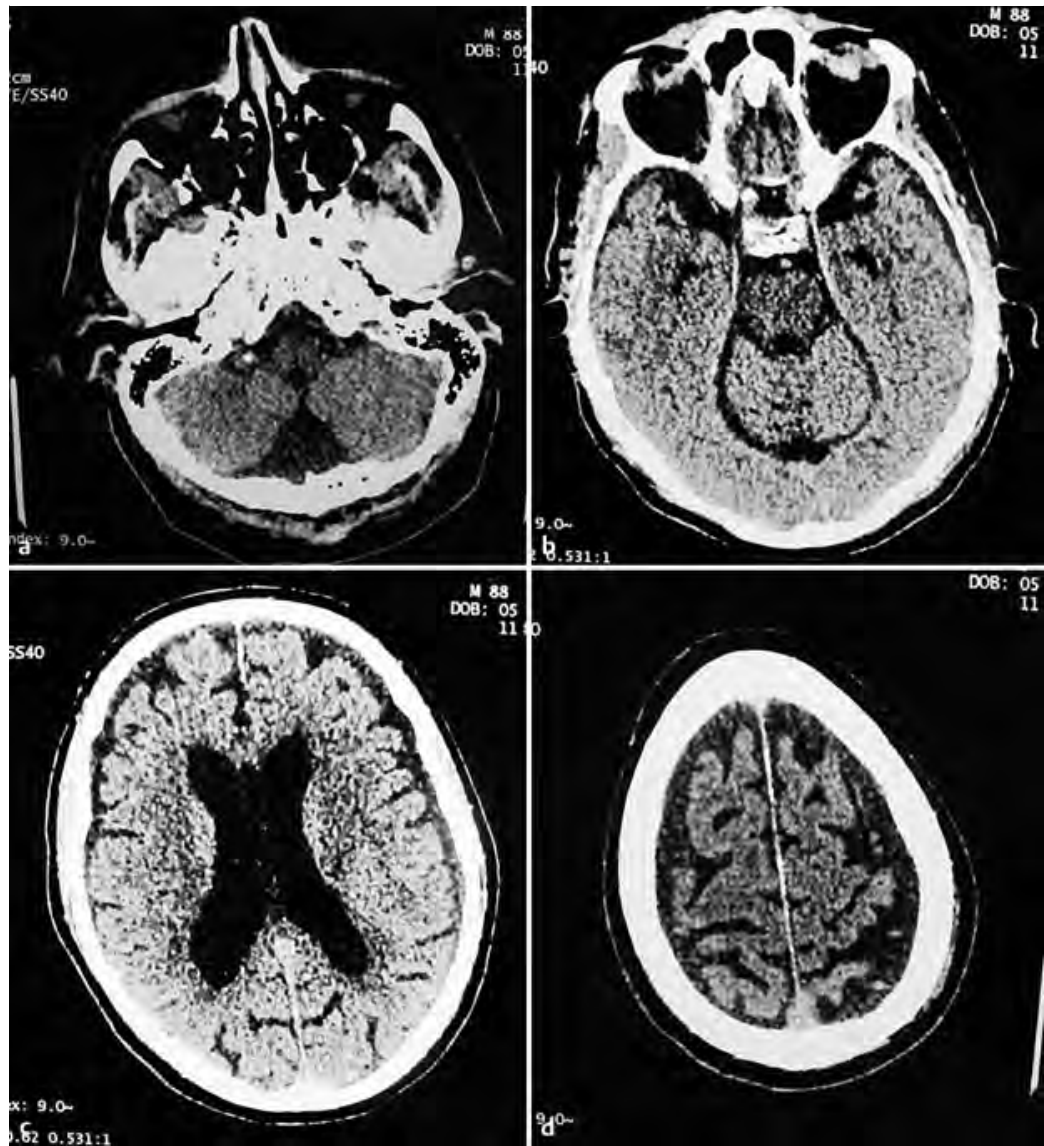

Abb. 25 Korrekte Lagerung des Patienten.

a-d Beispiel für axiale Schichten. 

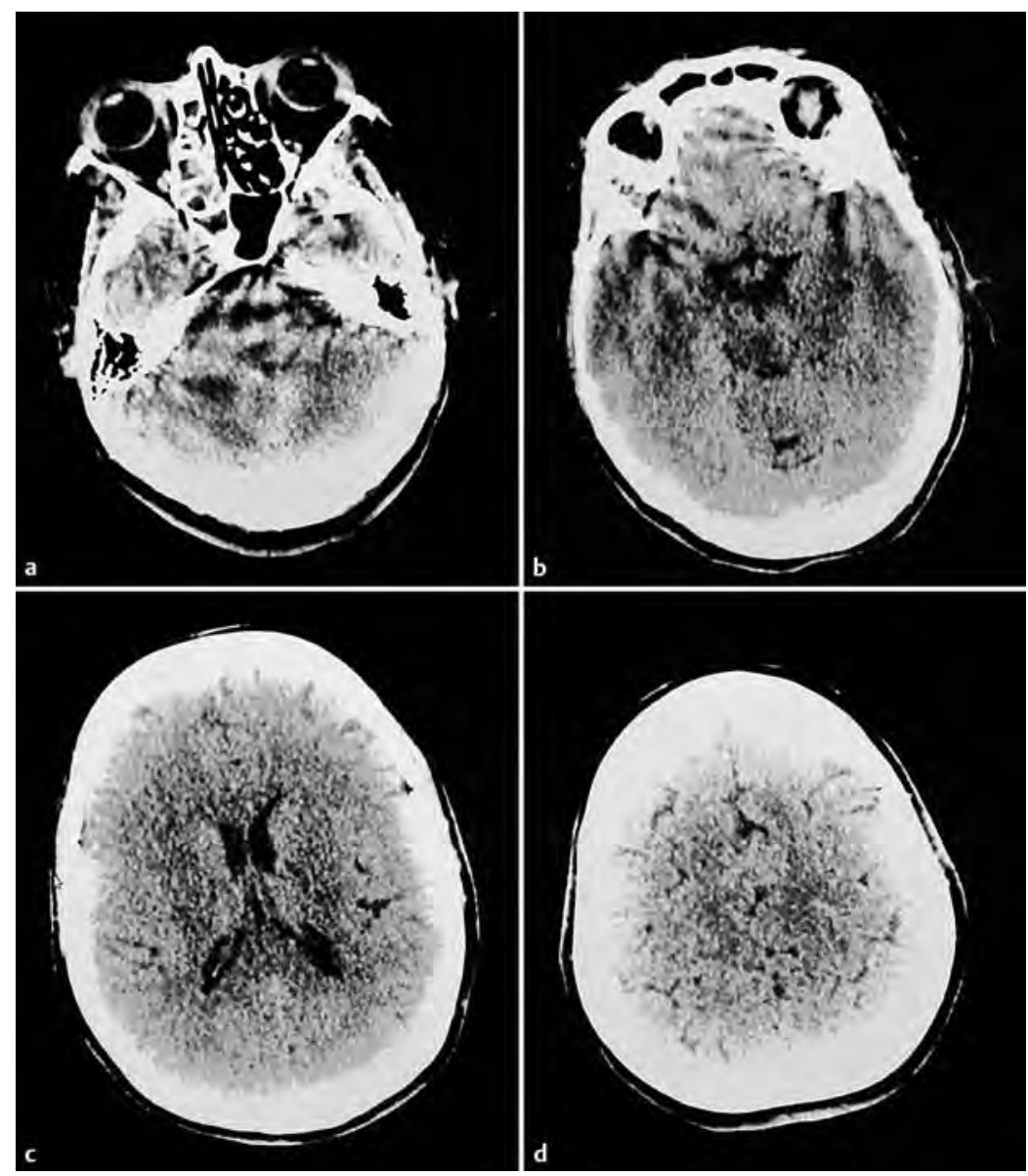

Abb.26 Untersuchung, bei der der Schädel nicht im Isozentrum gelagert werden konnte. a-d Beispiel für axiale Schichten.

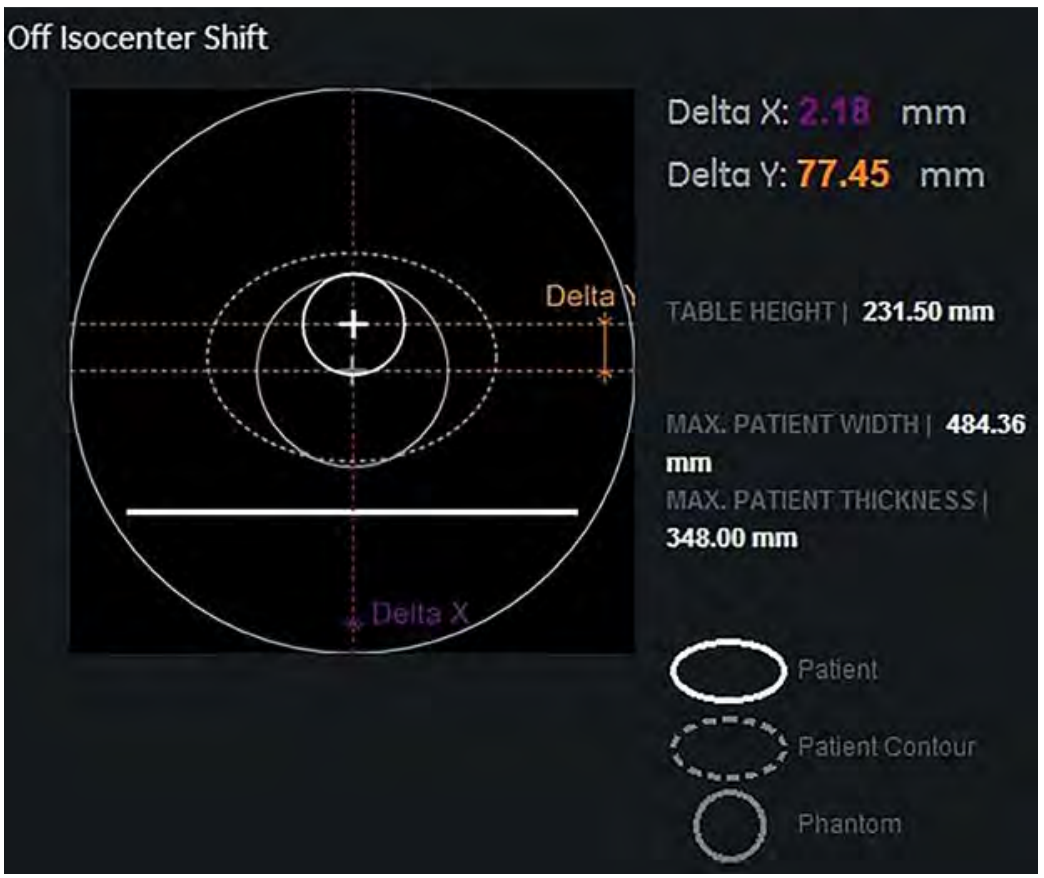

Abb.27 Darstellung der Patientenlagerung im Vergleich zum Isozentrum.

\section{Protokollparameter, Lagerung}

Bei Polytraumapatienten stellt die korrekte Lagerung im Gegensatz zu mobilen Patienten auf dem Patiententisch häufig ein Problem dar. Hier ist es wichtig, dass der zu untersuchende Bereich gut im Isozentrum gelagert wird, um eine optimale Bildqualität zu erreichen. Abb.25a-d zeigen eine Untersuchung nach Polytrauma, dargestellt ist nur der Schädel mit Standardparametern und korrekter Lagerung im Isozentrum.

- Abb.26a-d zeigen die Untersuchung, bei der der Schädel nicht im Isozentrum gelagert werden konnte. Aus diesem Grund wurden die Scanparameter angepasst (größeres Scan-FOV und höhere mAs-Werte, keine Dosismodulation). Die Bildqualität ist dadurch dementsprechend sehr viel schlechter bei viel höherer Strahlenbelastung! Mithilfe einer Dosissoftware ( $\bullet$ Abb.27) kann man nachträglich feststellen, ob der Untersuchungsbereich im Zentrum gelagert wurde. Man erkennt, dass der Patient nicht mittig, sondern zu hoch gelagert wurde. Dadurch ist er näher an der Röntgenröhre und wirkt für das System als kräftiger Patient, breiterer Kegelstrahl auf dem Detektor. Die Dosismodulation gibt mehr mAs ab, daraus folgt eine höhere Strahlenbelastung.

\section{Kernaussagen}

- Gute Patientenvorbereitung, Instruktionen und Lagerung helfen, Artefakte zu reduzieren.

- Es sollten patientenadaptierte Protokolle verwendet werden.

- Durch die Nutzung der iterativen Bildrekonstruktion sind eine Verbesserung der Bildqualität und eine starke Reduzierung der Dosis möglich.

- Der Anwender sollte das System regelmäßig kalibrieren und die Serviceintervalle einhalten. 


\section{Abstract \\ $\nabla$}

Computed tomography is an important part of medical diagnosis and therapy since its introduction in the 1970 s. The development and evolution in the early years led mainly to greater detail, higher resolution, and higher speed. As a result at the beginning of the 1990 s CT angiographies were possible with multislice CT whole-body scans. The progress in the IT sector also improved postprocessing techniques. Today the main focus is on the development of radiation protection. The claim is to produce the best diagnostic images using a dose as low as possible. But this medical examination technique is not completely free of image artifacts. This article looks at the different kinds of image noise and what the user can do to reduce or avoid them.

\section{Literatur}

1 Alkadhi $\mathrm{H}$ et al. Wie funktioniert CT? Berlin: Springer; 2011

2 Grillenberger A, Fritsch E. Computertomographie. Wien: Facultas Universitätsverlag; 2012

3 Kalender W. Computed Tomography. Erlangen: Publicis Corporate Pub; 2011

4 Prokop M, Galanski M. Spiral and Multislice Computed Tomography of the Body. Stuttgart: Thieme; 2003

\section{Über den Autor}

$\checkmark$

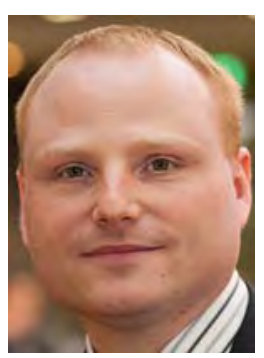

André Mayor

Jahrgang 1980. 1999 Abitur am Sophiengymnasium Colditz. 2003 Examen zum MTRA am Klinikum Nürnberg. 2003-2006 MTRA am Institut für Radiologie und Nuklearmedizin, Klinikum Pforzheim. 2006-2007 MTRA in der Praxis Fürth \& Fürth Land, Oberasbach. 2007-2013 MTRA am Institut für Radiologie, Spital Lachen. 2013 MTRA am Institut für Radiologie, Spital Limmattal. 2013/ 2014 leitender MTRA, RODIAG Diagnostic Center Zürich-Oerlikon, Zürich. Seit November 2014 Stellvertretung Röntgentechnik/IT und MTRA am Institut für Radiologie und Nuklearmedizin, Stadtspital Triemli, Zürich.

Interessenkonflikt: kein Interessenkonflikt angegeben

\section{Korrespondenzadresse}

André Mayor

Stadtspital Triemli

Departement für Transversale Disziplinen

Institut für Radiologie und Nuklearmedizin

Birmensdorferstrasse 497

8063 Zürich

Schweiz

Tel. $+41(0) 44 / 4663851$

Fax: $+41(0) 44 / 4663426$

E-Mail: andre.mayor@triemli.zuerich.ch

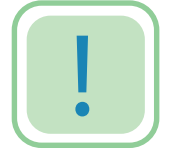

\section{Radiobonus}

Wer die CRTE/CPD-Fragen zu diesem Artikel bis zum 31. Oktober 2015 beantwortet, kann gewinnen: Wir verlosen 3 Exemplare des Werks Möller, Reif: CT- und MRT-Norisces malbefunde. 


\section{CRTE/CPD-Fragen Artefakte in der Computertomografie}

\section{1}

Wie kann man Ringartefakte minimieren?

A. Der CT sollte ausgeschaltet werden.

B. Eine regelmäßige Kalibrierung (mind. $1 \times$ pro Tag) sollte erfolgen.

C. Die Untersuchung mit verschiedenen Patientenlagerungen wiederholen.

D. Die Parameter auf $140 \mathrm{kV}, 300 \mathrm{~mA}$, Rotationszeit $1 \mathrm{~s}$ ändern.

E. Einen Bleischutz auf das Objekt legen.

\section{Wodurch lassen sich Pulsationsartefakte verringern?}

A. Die Gesamtscanzeit sollte erhöht werden.

B. Die Gesamtscanzeit sollte erniedrigt werden.

C. Es sollte Kontrastmittel verabreicht werden.

D. Der Röhrenstrom sollte verringert werden.

E. Keine der anderen Antworten trifft zu.

\section{Welches Implantat erzeugt die wenigsten Artefakte?}

A. Klammernähte

B. Hüftprothesen beidseits

C. Spondylodese

D. Fixateur extern

E. Knieprothese

\section{4}

Auf dem CT-Bild sind Aufhärtungsartefakte sichtbar.

\section{Was kann man für eine bessere Beurteilung machen?}

A. Man kann eine Nachrekonstruktion von dünneren Schichtbildern durchführen.

B. Man kann die Untersuchung wiederholen.

C. Man kann eine Nachrekonstruktion von dickeren Schichtbildern durchführen.

D. Man kann eine koronare Schichtebene akquirieren.

E. Keine der anderen Antworten trifft zu.

5 Welches stellt das optimale Rekonstruktionsinkrement bei einer Kollimation von 3,75 dar?

A. 0,312

B. $0,625-1,25$

C. 1,25

D. $1,9-3,5$

E. 5

\section{6}

A. kürzeren Bildrechenzeiten.

B. schlechterer Bildqualität.

C. höherer Patientendosis.

D. besserer Bildqualität.

E. Keine der anderen Antworten trifft zu.

7 Für das Postprocessing sollte der Datensatz möglichst
A. isobar sein.
B. isomer sein.
C. isodrink sein.
D. isotop sein.
E. isotrop sein.

8 Man ändert die gewählte Spannung von 120 auf $140 \mathrm{kV}$. Welche Antwort ist richtig?

A. Das Bildrauschen nimmt zu.

B. Der Kontrast nimmt zu.

C. Die Patientendosis nimmt ab.

D. Das Bildrauschen nimmt ab.

E. Die Untersuchung dauert länger.

9 Keinen Einfluss auf die Bildqualität beim Kardio-CT hat/ haben

A. die Herzfrequenz.

B. der Kreatininwert.

C. die Atemstellung.

D. eine sehr gute EKG-Ableitung.

E. Arrhythmien.

Welcher Untersuchungsparameter wird bei der Dosismodulation im CT automatisch an den Patienten angepasst?

A. Kontrastmittelmenge

B. Schichtdicke

C. Atmenanhaltedauer

D. Röhrenstrom

E. Rotationszeit 